\title{
Irrigation districts. Sustainable alternative for the economic development of Alto Humea-Meta ( Colombia)
} Distritos de riego. Alternativa
sustentable para el desarrollo económico
del Alto Humea-Meta (Colombia)'

\section{Ernesto Leonel Chávez Hernández ${ }^{2}$}

Juan Manuel Ochoa Amaya ${ }^{3}$

Luz Gladys Yarime Peña Ulloa ${ }^{4}$

Colombia
1 * Este artículo presenta una reflexión teórica a partir de la investigación adelantada por los autores en el Distrito de Riego del Humea. Proyecto financiado por la Universidad de los Llanos.

$2 \quad$ Magíster en Desarrollo Sostenible y Medio Ambiente (Universidad de Manizales), Economista (Universidad Católica de Colombia); Profesor de planta de la Universidad de los Llanos; correo electrónico Institucional: echavez@unillanos.edu.co. https://orcid.org/0000-00023008-0502

3 Doctor () en Estudios Territoriales (Universidad de Caldas), Magíster en Desarrollo Sostenible y Medio Ambiente (Universidad de Manizales), Especialista en Docencia Universitaria (Universidad Cooperativa de Colombia), Economista (Universidad Pedagógica y Tecnológica de Colombia U.P.T.C); Profesor de planta de la Universidad de los Llanos; correo electrónico Institucional: juan.ochoa@unillanos.edu.co. https://orcid.org/0000-00017198-0577

4 Magíster en Economía (Universidad de Manizales), Especialista en Gerencia del Recurso Humano (Universidad Jorge Tadeo Lozano), Economista (Universidad Pedagógica y Tecnológica de Colombia U.P.T.C); Profesora de tiempo completo de la Universidad de los Llanos; correo electrónico Institucional: yarime.pena@unillanos.edu.co. https://orcid.org/0000-0003-3628-6277

\section{RESUMEN}

El derecho al agua hace parte de la política pública en Colombia. El manejo del recurso, a través de distritos de riego, está favorecido con la ley 41 de 1993. Dicha ley fomenta la participación de los beneficiarios por medio de la transferencia para su administración (BID, 1995). Así a partir de que de 1976 se gestaron éstos en distintas partes de la geografía nacional como los de la Costa Atlántica, Valle del Cauca, Boyacá y algunos de menor envergadura distribuidos en todo el territorio colombiano. 
De otra parte, es función del Estado garantizar la construcción de infraestructura en función de la demanda. Así el desarrollo de estos proyectos se debate entre obras principales y secundarias de mano del Estado, mientras que las intraprediales van auxiliadas por el sector privado, los usuarios de distritos de riego. En el presente artículo éstos son examinados como una alternativa sustentable encaminada al desarrollo económico del Alto Humea analizando su viabilidad y sostenibilidad como objetivo general. Para ello a través de una revisión bibliográfica se analizaron diferentes teorías sobre valoración ambiental en relación con el agua, buscando una evaluación de la situación al tener en cuenta las diferentes categorías en relación con los precios sombra y las razones del precio cuenta del DNP. Finalmente se encontró que los indicadores socioeconómicos de los municipios son preocupantes dificultando el desarrollo óptimo de la calidad de vida así: $50 \%$ de la población cuenta con NBI, precariedad en los servicios públicos, analfabetismo y desplazamiento por conflicto armado.

PALABRAS CLAVE: Sostenibilidad; distritos de riego; recurso hídrico

\section{ABSTRAC:}

The right to water is part of public policy in Colombia. The management of the resource, through irrigation districts, is favored by Law 41 of 1993. This law encourages the participation of the beneficiaries through the transfer for its administration (IDB, 1995). Thus, since 1976 these were developed in different parts of the national geography as those of the Atlantic Coast, Valle del Cauca, Boyacá and some smaller distributed throughout the Colombian territory.

On the other hand, it is the function of the State to guarantee the construction of infrastructure according to the demand. Thus the development of these projects is debated between main and secondary works of the State, while the intraprediales are aided by the private sector, users of irrigation districts. In the present article these are examined as a sustainable alternative aimed at the economic development of Alto Humea, analyzing its viability and sustainability as a general objective. For this purpose, through a literature review, different theories on environmental valuation in relation to water were analyzed, seeking an evaluation of the situation when taking into account the different categories in relation to shadow prices and the reasons for the price of the DNP account. Finally, it was found that the socio-economic indicators of the municipalities are worrisome, hindering the optimal development of the quality of life as follows: $50 \%$ of the population has UBN, precariousness in public services, illiteracy and displacement due to armed conflict.

KEYWORDS: Sustainability; irrigation districts; water resources.

\section{INTRODUCCIÓN}

Colombia es un país que desde hace varios años ha estado avanzando en políticas orientadas a la regulación del uso del agua para riego (Ley 41 de 1993) que buscan además una participación de los beneficiarios. A este respecto, este País ha sido pionero en lo referente a la transferencia de distritos de riego a los usuarios para su administración (BID, 1995). En efecto, se han entregado distritos en la Costa Atlántica, Tolima, el Valle del Cauca, Boyacá y pequeños distritos en casi todo el territorio nacional.

No obstante, las nuevas condiciones han determinado una nueva forma de administrar las aguas habida cuenta de que el Estado es el propietario de los recursos naturales no renovables, según la Constitución Política de Colombia, y debe planificar su manejo y aprovechamiento para garantizar su conservación, restauración o sustitución, (Art. 80). En cuanto a los distritos de riego, el 
Estado se encarga aquí de la construcción de infraestructura que incluye obras principales, secundarias e intraprediales. Las dos primeras, generalmente se ejecutan con cargo al Estado y las intraprediales por ley deben asumirlas los usuarios de acuerdo con lo establecido en la Ley 41 de 1993. No obstante, la ley se tradujo en un encarecimiento exagerado del costo de las inversiones y una concentración bastante discutible en la asignación de los subsidios, entre otras cosas asignándolos en contravía del espíritu de la misma ley (Ramírez, 1998).

En lo que tiene que ver con el manejo de los distritos Colombia comenzó su transferencia a los usuarios desde 1976 de manera que hay una larga trayectoria comparativamente con otros países en esta materia. Sin embargo, en el Río Humea, unos propietarios de terrenos no esperaron la intervención del Estado y han adelantado algunas obras de adecuación mediante el desvío del cauce lo que se presume ha causado considerables efectos ambientales. Por esto se busca hacer una evaluación económica social y ambiental de tales obras, analizar su viabilidad y sostenibilidad hacia el futuro y comparar los resultados con un distrito de similares características construido por el sector público, esto se hará, mediante un trabajo de campo para la información relacionada con uso del suelo, área, producción y rendimientos; para el análisis comparativo se tomarán como costos de referencia los estimados del distrito Ariari, cuyos diseños están en el INCODER.

Tomando en consideración que los recursos naturales deben ser aprovechados de forma sostenible, la propuesta que aquí se plantea consiste en evaluar los costos y beneficios ambientales del actual aprovechamiento del Río Humea que se ejecuta por medio de la Asociación Agropecuaria de Usuarios del Río Humea Asohumea, a partir de una experiencia muy particular en Colombia si se tiene en cuenta que es un distrito que en 2011 cuenta con unas
15000 hectáreas regadas con inversión completamente privada y sin intervención del Estado.

La investigación aportará un documento que sirva para el análisis de la inversión pública en distritos de riego que permita comparar costos y beneficios entre el sector público y privado.

\section{PLANTEAMIENTO DE LATEMÁTICA}

En Colombia las aguas para riego son otorgadas por concesión mediante un acto administrativo de las Corporaciones Autónomas Regionales (CAR), encargadas de vigilar su uso, distribución y aprovechamiento. Con base en estas consideraciones, desde mediados de 1990 se han iniciado algunas acciones orientadas principalmente por el Banco Mundial para optimizar el uso eficiente de los recursos hídricos incluyendo una revisión en las tarifas que se cobran por al uso del agua ${ }^{5}$.

La valoración del agua es una preocupación reciente, se han adelantado estudios que proponen terminologías nuevas como "agua virtual" o "waterpolicy" (Waningen Agriculture University - The World Bank. 1999). En este sentido existen distintas metodologías para la evaluación social de proyectos, como los precios sombra entre otras. En relación con ello cabe mencionar a Partha Dasgupta y Amartya Sen (1973) que publicaron una metodología para evaluación de proyectos de inversión, al igual que otros como Londero y Little-MirrleesSquire-Van der Tak, que dieron nombre a la metodología LMST.

Algunos estudios han advertido sobre la insuficiencia de los precios de mercado para medir todos los aspectos relacionados con las actividades económicas de la humanidad de ahí los precios sombra, que ponderan costos y

$5 \quad$ A este respecto véanse los informes del Banco Mundial sobre los créditos 3113 y 2667. 
beneficios a precios de mercado para algunos factores (Dasgupta y Sen, 1973).

Igualmente, los costos ambientales han sido estudiados por Gudynas (1997), Macve, Arnold y Bailey (1997), Rosegrant, Cai y Cline (2002) proponiendo metodologías alternativas para medición, incluso fue publicado un documento (2002) ${ }^{6}$ que toma en consideración los costos del agua, riego, producción de alimentos, la sostenibilidad y las implicaciones hacia el futuro de las actuaciones presentes. Este documento plantea distintos aspectos para una valoración del agua, señalando explícitamente lo siguiente:

El desarrollo hídrico es crítico para la seguridad alimentaria en muchas regiones del mundo. Actualmente, 250 millones de hectáreas bajo riego alrededor del mundo, es cerca de cinco veces la superficie regada a comienzos del siglo $X X$. Sin riego, el incremento en los rendimientos agrícolas y los productos que han de alimentar el crecimiento de la población mundial no será posible (Rosegrant, 2002, p.1).

A ello agregan los expertos que "la agricultura bajo riego es el usuario principal del agua, que explica, por ejemplo, el 80 por ciento del consumo hídrico global y el 86 por ciento del consumo de agua en los países en desarrollo en 1995" (Rosegrant, 2002, p.1). Estas cifras tienen importantes repercusiones económicas si se tiene en cuenta que, en algunos países del mundo, regar una hectárea puede costar hasta US\$8 300 (Rosegrant, 2002) y que "al paso que vamos, aún sin que Colombia soporte las desastrosas sequías del África, en breve lapso no va a haber mucha diferencia entre los costos de construir distritos en el Sahara o en cualquier parte de nuestra geografía" (Ramírez, 1998. p.183).

6 ROSEGRANT, Mark. W., XIMING, Cai y CLINE, Sarah A. World Water and Food to 2025. Dealing with Scarcity. International Policy Research Institute. Washingto D.C.
La economía del agua es un asunto que ha llamado la atención de muchos investigadores y la literatura sobre este particular es abundante. Entre los documentos más completos consultados, un estudio afirma que hay una sobreexplotación de agua subterránea y menciona los casos de India, China, Estados Unidos, Norte de África y la Península Arábiga con un volumen aproximado de 160 mil millones de metros cúbicos al año 7 .

Si se incluyera el resto del mundo -dice el estudio- y el volumen creciera $25 \%$-un supuesto no irreal- el déficit mundial de agua ascendería a 200 mil millones de metros cúbicos al año. La gran mayoría de esta sobreexplotación de aguas subterráneas es usada para granos bajo riego que indica que unas 180 millones de toneladas de grano -alrededor del 10 por ciento de la producción mundialestán siendo producidas mediante el agotamiento de fuentes hídricas: $\mathrm{Si}$ tal proporción de la agricultura está operando en condiciones deficitarias de agua actualmente, ¿dónde se va a encontrar el agua adicional para riego que se necesita para satisfacer las demandas de alimentos de más de 2000 millones adicionales de personas que se esperan hacia el año 2030? (Postel, 1999, p.80)

En adición a lo anterior no solamente el crecimiento vegetativo de la población y las necesidades crecientes e infinitas del ser humano tienen que ver con la agricultura bajo riego, también lo es la búsqueda de rendimientos

$7 \quad$ En la versión norteamericana consultada, se habla de "160 billion cubic meters a year" pero el concepto de billón en inglés americano equivale a mil millones en español. De ahí la cifra mencionada.

$8 \quad$ Pillar of sand. Can the irrigation miracle last? Worldwatch books. New York. 1999.. Traducción no official. 
agrícolas como fuente de contaminación pues, de acuerdo con el Instituto Mundial de Recursos (WRI), alrededor del 66 por ciento de la tierra agrícola regada ha sido degradada por diferentes grados de erosión, salinización, agotamiento de nutrientes, compactación, degradación biológica, o polución durante los últimos 50 años (World Resources Institute, 2001, p.124).

Así es que la evaluación ambiental del distrito de riego del río Humea debe hacerse a la luz de los desarrollos teóricos que han incorporado los costos de la degradación de las fuentes y los efectos que el riego tiene sobre los suelos en términos de erosión y salinización, además de valorar el recurso mismo. Por ejemplo, para una simulación de evolución de los costos del agua en $2002^{9}$, se utilizaron como precios de referencia de un metro cúbico de agua 0.46 dólares para el África subsahariana y 0.77 para los Estados Unidos de América, lo cual es un precio bastante aproximado si se compara con lo que en Colombia se cobra por esa misma unidad de consumo urbano y teniendo en cuenta la revaluación del peso colombiano ${ }^{10}$.

El distrito de riego distribuye agua para diferentes actividades económicas del agro como la avicultura, piscicultura, ganadería y producción primaria con valor agregado, las cuales ocupan 60 predios, aproximadamente más de 40.000 hectáreas. Éstas se dividen en 3 zonas comprendidas desde el rio Upía hasta el rio Cabuyarito (zona 1); rio Cabuyarito al rio Humea (zona 2); y rio Humea al municipio de Cumaral (zona 3).

$9 \quad$ Ver Rosegrant. Table 6.1 Pág. 140.

$10 \quad$ Tomando en consideración que en 2002 la tasa de cambio era del orden de $\$ 2500$ por dólar, el costo por metro cúbico oscilaría entre $\$ 1200$ y $\$ 2000$ que estaría dentro de parámetros razonables si se tiene en cuenta que en Bogotá un metro cúbico de agua en enero de 2011 se cobraba a $\$ 2845$, para consumo humano en áreas urbanas.

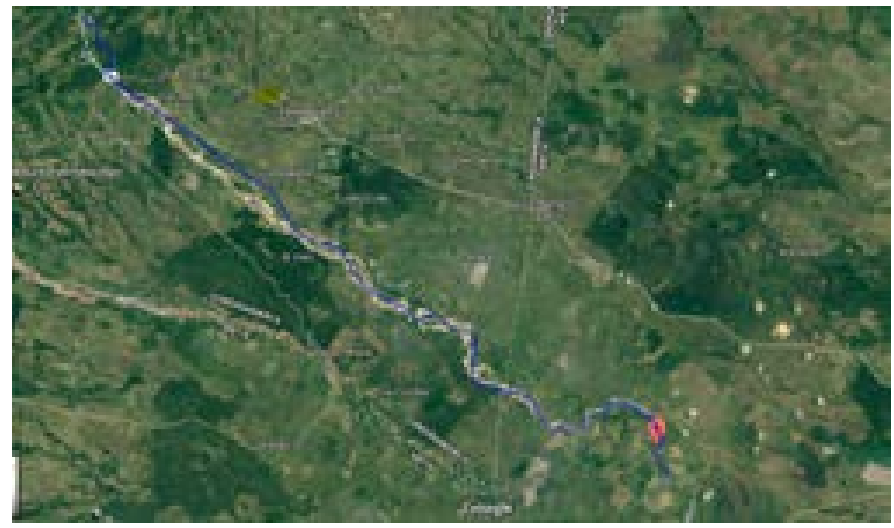

Figura 1. Zona de estudio

Elaboración propia a través de Google earth. (c.2014). "Obtenido de US Dept of State

Geographer Data SIO, NOAA, U.S. Navy, NGA, GEBCO image LandSat 2014 Google".

\section{DESARROLLO}

\subsection{Costos y beneficios económicos y sociales de las actividades representativas en el distrito de riego}

El Meta tiene un evidente rezago en la implementación de los distritos de riego, siendo el porcentaje de Unidades de Producción Agropecuaria (UPA) inferior al de otros departamentos. Situación evidenciada en los sistemas de riego que oscilan entre un 10,1\% a $20 \%$ UPA, mientras que Amazonas y Guainía que tienen más de $30 \%$ de UPA. Adicionalmente el porcentaje de UPA con sistema de riego por aspersión es uno de los más bajos del país, ubicándose de $0,1 \%$ a $2 \%$ UPA para el Meta y Huila (DANE, 2016).

De otra parte, en el Censo Nacional Agropecuario (CNA) evidencia que el departamento del Meta se ve beneficiado por sus recursos naturales, por ello el acceso al agua medido en porcentaje de UPA es de $81,1 \%$ a $90 \%$, superado por Caquetá y Bolívar con un acceso de más del $90 \%$ de las UPA. Así es que existe una correlación con el uso del agua y con el cuidado de esta, 
y los departamentos que tienen más acceso a este recurso lo preservan más como Caquetá y Vichada con más del $70 \%$ de las UPA y el Meta junto con el Casanare, con un $53,1 \%$ a $70 \%$ de las UPA (Fuente: Elaboración propia, con base en el Libro 3Cer CNA 2016 tomo 1, 2 y 3)

De otra parte, el Meta se ha ubicado en un nivel medio bajo con respecto al porcentaje de UPA teniendo dificultades por: contaminación 7,1\% a $15 \%$ UPA, sequía $25,1 \%$ a $60 \%$ UPA, $10,1 \%$ A $24,1 \%$ (Fuente: Elaboración propia, con base en el Libro 3Cer CNA 2016 tomo 1, 2 y 3) en comparación con otros departamentos.

De acuerdo con el INPARME S.A. (2010) la producción más representativa del año 2009 fue la de aceite de palma con 5338 toneladas de aceite, mientras que la producción de arroz fue baja en comparación con otros cultivos, siendo su participación la mostrada en la tabla 1 así:

\section{Tabla 1.}

Participación en el área sembrada para el primer semestre, por sistema, en los departamentos de los Llanos Orientales, 19992011.

\begin{tabular}{ccc}
\hline Año & Riego & Secano \\
\hline 1999 & $27 \%$ & $73 \%$ \\
2001 & $33 \%$ & $67 \%$ \\
2003 & $32 \%$ & $68 \%$ \\
2004 & $26 \%$ & $74 \%$ \\
2005 & $23 \%$ & $77 \%$ \\
2006 & $23 \%$ & $77 \%$ \\
2007 & $27 \%$ & $73 \%$ \\
2008 & $22 \%$ & $78 \%$ \\
2009 & $22 \%$ & $78 \%$ \\
2010 & $17 \%$ & $83 \%$ \\
2011 & $16 \%$ & $84 \%$ \\
\hline
\end{tabular}

Fuente (FEDEARROZ, 2011)

Los rendimientos dependen de la época del año en la cual se cultive, así en arroz paddy a nivel departamental el rendimiento promedio se puede observar en la tabla 2.

Tabla 2.

Rendimientos en toneladas de paddy seco para el segundo semestre, por sistema. Llanos

Orientales, 1999-2010 comparativo

\begin{tabular}{ccccr}
\hline \multirow{2}{*}{ AÑO } & \multicolumn{2}{c}{ META } & \multicolumn{3}{c}{ CASANARE } \\
\cline { 2 - 5 } & Riego & \multicolumn{2}{c}{ Secano } & \multicolumn{2}{c}{ Riego } & Secano \\
\hline \multicolumn{4}{c}{ Toneladas paddy seco / hectárea } \\
\hline 1999 & 4,77 & 4,68 & 4,51 & 4,26 \\
\hline 2000 & 4,77 & 4,41 & 4,74 & 4,59 \\
\hline 2001 & 4,86 & 4,52 & 4,85 & 4,55 \\
\hline 2002 & 4,72 & 4,56 & 5,19 & 4,76 \\
\hline 2003 & 4,92 & 4,73 & 4,63 & 4,41 \\
\hline 2004 & 5,52 & 4,37 & 3,91 & 3,97 \\
\hline 2005 & 4,75 & 4,46 & 4,2 & 3,62 \\
\hline 2006 & 4,76 & 4,62 & 5,07 & 4,96 \\
\hline 2007 & 4,82 & 4,8 & 4,79 & 4,45 \\
\hline 2008 & 4,9 & 4,83 & 4,81 & 4,51 \\
\hline 2009 & 4,68 & 4,41 & 4,16 & 4,39 \\
\hline 2010 & 4,66 & 4,6 & 4,72 & 4,6 \\
\hline
\end{tabular}

Fuente: (FEDEARROZ, 2011)

Los costos del cultivo de arroz vienen establecidos por Fedearroz en varios aspectos, entre ellos: asistencia técnica, arriendo, preparación, riego, fertilizantes, protección, recolección, transporte, otros (FEDEARROZ, 2011). 
Tabla 3.

Variaciones porcentuales para cada aspecto que constituye el costo real de la producción de arroz.

\begin{tabular}{|r|r|r|r|r|r}
\hline & Arriendo & $\begin{array}{l}\text { Preparació } \\
n\end{array}$ & $\begin{array}{l}\text { Fertilizante } \\
\text { s }\end{array}$ & Protección & Total \\
\hline $\mathbf{2 0 0 0}$ & $0,40 \%$ & $-6,50 \%$ & $11,60 \%$ & $2,90 \%$ & $-3,40 \%$ \\
\hline $\mathbf{2 0 0 1}$ & $-7,30 \%$ & $0,10 \%$ & $2,20 \%$ & $9,90 \%$ & $4,40 \%$ \\
\hline $\mathbf{2 0 0 2}$ & $4,60 \%$ & $7,90 \%$ & $0,80 \%$ & $-10,30 \%$ & $-1,90 \%$ \\
\hline $\mathbf{2 0 0 3}$ & $5,70 \%$ & $-2,60 \%$ & $-1,30 \%$ & $14,10 \%$ & $3,80 \%$ \\
\hline $\mathbf{2 0 0 4}$ & $3 \%$ & $6,20 \%$ & $12,50 \%$ & $-1,80 \%$ & $6,50 \%$ \\
\hline $\mathbf{2 0 0 5}$ & $-15,70 \%$ & $0,50 \%$ & $-11,50 \%$ & $-4,80 \%$ & $-7,1$ \\
\hline $\mathbf{2 0 0 6}$ & $24,40 \%$ & $-0,30 \%$ & $-6,60 \%$ & $6,60 \%$ & $1,70 \%$ \\
\hline $\mathbf{2 0 0 7}$ & $3 \%$ & $-0,20 \%$ & $19,80 \%$ & $-21,70 \%$ & $-6,80 \%$ \\
\hline $\mathbf{2 0 0 8}$ & $9,80 \%$ & $2,80 \%$ & $85,20 \%$ & $0,4 \%$ & $15,80 \%$ \\
\hline $\mathbf{2 0 0 9}$ & $47,50 \%$ & $28,20 \%$ & $2 \%$ & $11,40 \%$ & $15,70 \%$ \\
\hline $\mathbf{2 0 1 0}$ & $-10,70 \%$ & $0 \%$ & $-30,50 \%$ & $-14,90 \%$ & $-11,20 \%$ \\
\hline
\end{tabular}

Fuente: (FEDEARROZ, 2011)

Lo anterior indica la variación anual de los precios del arroz para el período analizado (2000 - 2011), así como las alzas y bajas en los precios cada vez son más pronunciadas (sobre reaccionando), evidenciando así la inestabilidad de las variables asociadas al precio, como el área sembrada y los inventarios. Igualmente, la línea de tendencia deja ver que los ciclos alcistas y bajistas de los precios cada vez acortan su distancia, es decir, que los picos tienden a presentarse con mayor frecuencia en comparación de lo que ocurría en la década anterior. Para los Llanos Orientales el comportamiento de los precios es similar principalmente por el cálculo del coeficiente de correlación de los precios del paddy verde de Casanare (Yopal) y el Meta (Villavicencio) que arroja un valor de $98,9 \%$. Lo anterior quiere decir que si el precio de Villavicencio se incrementa en cualquier porcentaje, el precio de Yopal tiene un movimiento similar en la misma dirección (FEDEARROZ, 2011).

En cuanto a los beneficios sociales ASOHUMEA utiliza el recurso hídrico para el abastecimiento de diferentes actividades agropecuarias, buscando garantizar el mantenimiento frecuente del cauce y un control de mitigación de los impactos ambientales de la zona en la que tiene operación el distrito de riego, asimismo recoge recursos por el uso del agua, lo cual debe generar, en teoría, beneficios sociales para aquellos que usan el recurso en el área de influencia, y en general a todos los stakeholders que directa $o$ indirectamente tengan relación con el ejercicio económico. El uso del agua por predio y litros por segundo es: 
Tabla 4.

\begin{tabular}{|l|r|l|r}
\hline PREDIO & $\begin{array}{l}\text { DOTACIÓN } \\
\text { L/SEG }\end{array}$ & PREDIO & \multicolumn{2}{l}{$\begin{array}{l}\text { DOTACIÓN } \\
\text { LSEG }\end{array}$} \\
\hline San José & 30 & Las leonas & 100 \\
\hline Porfía & 200 & Caporales & 450 \\
\hline Aposentos & 200 & La guaca & 250 \\
\hline Playa Rica & 600 & Macapay & 200 \\
\hline $\begin{array}{l}\text { Nagui T. } \\
\text { Caĥos }\end{array}$ & 200 & La Carolina & 160 \\
\hline $\begin{array}{l}\text { Sabana de } \\
\text { Guio }\end{array}$ & 300 & Cañadas & 645 \\
\hline Santa Martha & 85 & San Miguel & 450 \\
\hline San Juanito & 700 & Guampay & 354 \\
\hline Campo Alegre & 500 & Guavijo & 250 \\
\hline El Edén & 200 & Corocora & 250 \\
\hline La Reserva & 500 & Carpintero & 250 \\
\hline Pernambuco & 200 & La misión & 700 \\
\hline La Gran Diana & 900 & El Boral & 640 \\
\hline Yalconia & 900 & & \\
\hline Casa Brava & & & \\
\hline
\end{tabular}

El uso del agua por predio, por litros por segundo.

Fuente: Mapa de la Asociación Agropecuaria De Usuarios Del Río Humea "ASOHUMEA". V/ cio. Meta. Colombia. 2007 tomado de (Guzmán Ramírez, 2010)
Lo anterior indica el uso del agua de los predios que conforman el distrito de riego, lo cual demuestra el control que se le hace al recurso hídrico, por consiguiente, promueve el desarrollo integral de la región que, junto con la evaluación de impacto ambiental, forma parte integral de la actividad del distrito. (Guzmán Ramírez, 2010) Estado ambiental de los municipios Cumaral, Medina, Paratebueno, Barranca de Upía y Cabuyaro:

Todos los municipios mencionados arriba a excepción de Medina, hace 10 años tenían un gran daño ambiental asociado a deforestación, contaminación de ríos y desatención social. Las causas de la deforestación han sido mecánicas y también se ha presentado omisión del mantenimiento de las cuencas de los ríos. Actualmente ASOHUMEA trabaja de la mano con sus asociados en la reforestación de las cuencas.

En la tabla 5 se relacionan los predios que cultivan palma en el distrito de riego, resaltando en especial las distancias, el área de los predios, el aporte de cada predio y la relación precio/ hectárea. 


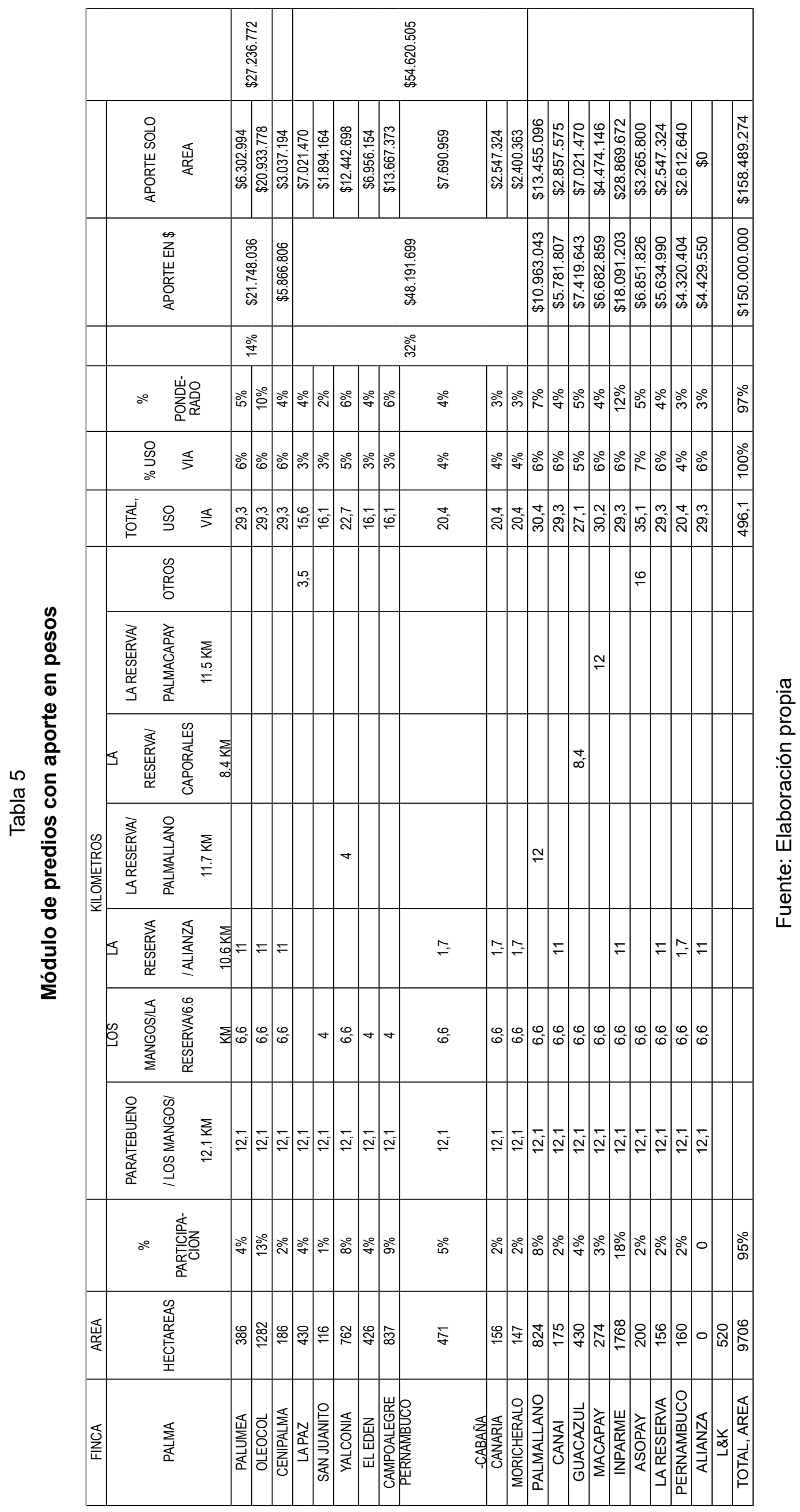


Con la anterior información se evidencia una correlación en el porcentaje de la participación y las hectáreas de cada predio, asimismo el aporte monetario por predio, destacando principales distancias de Paratebueno/ los mangos, los mangos a reserva con $12,1 \mathrm{~km}$ y $6,6 \mathrm{~km}$ respectivamente.

Tabla 6. Distancia en Kms al distrito de riego por predio.

\begin{tabular}{|c|c|c|c|}
\hline No. & DESDE & HASTA & DISTAN. KMS. \\
\hline 1 & PARATEBUENO & PALUMEA & 2,7 \\
\hline 2 & & CENIPALMA & 3,8 \\
\hline 3 & & CARIMAGUA & 6,2 \\
\hline 4 & & LOS MANGOS & 12,1 \\
\hline 5 & LOS MANGOS & CAMPOALEGRE & 2,2 \\
\hline 6 & & EL. EDEN & 5,9 \\
\hline 7 & & CRUCE LA RESERVA & 6.6 \\
\hline 8 & & PREDIO LA PAZ & 3,5 \\
\hline 9 & CRUCE LA RESERVA & COAGRES & 0,5 \\
\hline 10 & & CRUCE PALMALLANO & 6,1 \\
\hline 11 & & CAPORALES & 8,4 \\
\hline 12 & & PALMACAPAY & 11,5 \\
\hline 13 & & GUACAZUL & 13 \\
\hline 14 & & CANAI & 18,9 \\
\hline 15 & & PALMALLANO & 11,7 \\
\hline 16 & & PERNAMBUCO & 1,7 \\
\hline 17 & & OLEOCOL & 2,9 \\
\hline 18 & & GRAN DIANA & 3,5 \\
\hline 19 & & ALHUMEA & 10,6 \\
\hline 20 & & CRUCE INPARME & 11,2 \\
\hline 21 & & LA MISION & 12,5 \\
\hline 22 & & LA HORQUETA & 16,4 \\
\hline 23 & PALUMEA & ALHUMEA & 27,5 \\
\hline 24 & CARIMAGUA & ALHUMEA & 22,9 \\
\hline 25 & CENIPALMA & ALHUMEA & 24,4 \\
\hline 26 & CAPORALES & CRUCE GUARUPAY & 13,6 \\
\hline 27 & LA HORQUETA & YARICO & 1,3 \\
\hline 28 & & CRUCE GUARUPAY & 4,1 \\
\hline 29 & & LA EMBALADA & 11,4 \\
\hline 30 & & PUENTE RIO HUMEA & 13,7 \\
\hline
\end{tabular}

Fuente: Elaboración propia 
En la tabla se observa que en promedio la distancia desde diferentes puntos del distrito de riego hasta los diferentes predios que lo usan se encuentra en 9,3 km lo cual indica que a pesar de que ponderar no revela un indicador certero, se puede concluir que en general la distancia es alta y que el distrito surte a zonas donde es difícil el acceso al recurso hídrico.

La influencia del distrito de Riego del Rio Humea, exige indagar en las prácticas de protección de suelos y aguas, ya que de acuerdo con el instituto mundial de recursos (WRC), por lo menos el $66 \%$ de las tierras regadas han sido afectadas en diferentes grados de erosión, salinización y pérdida de nutrientes.

Con respecto a lo anterior para contrarrestar estos efectos algunos productores han realizado significativamente algún tipo de práctica de protección del suelo, mediante la labranza mínima, siembra sin remoción de suelos, siembra de cobertura vegetal, rotación de cultivos entre otros, aun así la gran mayoría no realiza ningún tipo de protección del suelo, lo mismo sucede con las prácticas de protección del agua, ejecutan alguna medida para contrarrestar la disminución y detrimento en este recurso. Si bien un significante grupo de productores ejecutan alguna practica de protección del suelo y del agua, es innegable que en futuro los rendimientos productivos y beneficios económicos en el municipio se vean comprometidos pues el deterioro que en los suelos y cuenca hidrográfica podrían hacerse presentes.

Dadalaimportanciadel rioHumeaparalaactividad agropecuaria, su preservación ha exigido la creación de la comisión conjunta encargada del manejo de su cuenca, integrada por la dirección de acción integral de recursos hídricos del ministerio de ambiente, las corporaciones autónomas regionales CORPOGUAVIO, CORMACARENA, CORPORINOQUIA, además de organizaciones privadas y comunitarias que complementas este consejo para el diseño del Plan de Ordenamiento y Manejo de Cuencas Ambientales POMCA del rio Humea.

La utilización de la cuenca hídrica con fines agropecuarios por sí solo no supone un problema directamente, dichas actividades constituyen un instrumento que garantiza a los pobladores del área rural una fuente de ingresos para la satisfacción de sus necesidades, pero en medio de dicha labor el manejo de prácticas y cultivos cuestionados y denunciados -más no ilícitos- por organizaciones sociales, constituyen el verdadero problema, pues no se debe considerar ni planear el desarrollo integral sin incluir en medio de su estudio las necesidades $\mathrm{y}$ atenciones del hombre y la naturaleza como agentes vulnerables ante la influencia del mercado, con lo cual es necesario la reconversión de valores que permita generar equilibrio entre los actores interesados en el uso de prácticas y la sustentabilidad de los recursos y medios bióticos del entorno.

\section{CONCLUSIONES}

Los municipios cuentan con indicadores socioeconómicos preocupantes, lo que dificulta el desarrollo óptimo de la calidad de vida de las personas.

En cuanto al manejo de los recursos ambientales, se evidencia que no existe homogeneidad en las leyes y normas que se dictan de las diferentes instituciones encargadas para tal fin, como lo son las Corporaciones Autónomas Regionales, Secretarias de Ambiente y el Ministerio de ambiente.

Cabe resaltar que Asohumea administra el recurso hídrico y promueve prácticas ambientales positivas, no es posible dejar a un lado uno de los aspectos más relevantes que tiene el distrito, en comparación a sus homólogos en el resto del país, y es que su origen, desarrollo, administración y actual funcionamiento es 
financiado por recursos privados, lo que genera que uno de sus objetivos sea generar ganancias lo cual contradice que sea una sociedad sin ánimo de lucro.

Los resultados arrojan un crecimiento en el nivel de hectáreas sembradas, para los diferentes cultivos que funcionan contiguos al distrito de riego, adicionalmente según información de ASOHUMEA existen proyectos encaminados en el mejoramiento de los impactos ambientales que generan las actividades económicas.

\section{REFERENTES BIBLIOGRÁFICOS}

Banco Mundial. (1991). Libro de consulta para Evaluación Ambiental.

Barranca de Upía. (S.F). Barranca de Upía Meta. Obtenido de www.barrancadeupia meta.gov.co/indicadores_anuales. shtml?apc=bexx-1-\&x=2137585

Cabuyaro. (S.F). CABUYARO - META. Obtenido de http://www.cabuyaro-meta.gov.co/ informacion_general.shtml\#economia

Cumaral. (S.F). Cumaral - Meta. Obtenido de http://www.cumaral-meta.gov.co/ informacion_general.shtml

DANE. (2016). Censo Nacional Agropecuario. Bogortá: GIT Área de Comunicación

Environment., A. a. (1991). U.S. DEPARTMENT OF AGRICULTURE.

GUDYNAS, Eduardo. Ecología, mercado y desarrollo. Políticas ambientales, libre mercado y alternativas. Vintén Editores. 2da. edición, Instituto Ecología Política, Santiago de Chile. 1997.

MACVE, Richards. Accounting for environmental cost. In: Richards, Deanna, (ed.) The industrial green game: Implications for environmental design and management. National Academy Press, Washington, USA, pp. 185-199. (1997).
Medina. (S.F). Medina - Cundinamarca. Obtenido de http://www.medinacundinamarca.gov.co/informacion general.shtml\#economia ojo no está dentro del texto.

Ministerio de agricultura y desarrollo territorial. (25 de enero de 1993). LEY 41 DE 1993. Obtenido de Diario Oficial No. 40.731.: https://www.minagricultura.gov. co/Normatividad/Leyes/Ley $\% 2041 \% 20$ de\%201993.pdf

Paratebueno. (S.F). Paratebueno Cundinamarca. Obtenido de http://www. paratebueno-cundinamarca.gov.co/ informacion_general.shtml

Postel, S. (1999). Pillar of Sand: Can The Irrigation Miracle Last?

Ramirez, M. Á. (1998). Análisis de la política de adecuación de tierras en Colombia. En Planeación y desarrollo. Revista del Departamento Nacional de Planeación. Vol. XXIX. Núm. 2. Bogotá.

Rosegrant, M. W. (2002). World Water and Food to 2025. Washington D.C. 